mRNA in neointima from day 5 to day 14 . At day 21, compared with no Electroporation-mediated group, no transfection group and GFP transfection group, AT2R transfection reduced $\mathrm{I} / \mathrm{M}$ intimal/medial area ratio significantly $(0.85 \pm 0.1,1.32 \pm 0.19,1.51 \pm 0.19,1.49 \pm 0.25$, $\mathrm{p}<0.01)$. No significant difference between no Electroporationmediated group, GFP group and no transfection group was observed. Conclusion The results of this study provide evidence that electroporation is an effective means for introducing naked AT2R DNA into the blood vessel wall and gene transfer of AT2R in vessel wall may effectively inhibit VSMC proliferation and neointimal hyperplasia in the rat carotid arteries after balloon angioplasty.

\section{e0054 RECOMBINANT ADENO-ASSOCIATED VIRUS SEROTYPE 9 TRANSFECTION OF RATS H9C2 CELLS IN VITRO}

doi:10.1136/hrt.2010.208967.54

Gao Xia, Ma Yi-Tong, Yang Yi-Ning, Xiang Yang, Chen Bang-Dang, Liu Fen. Department of Cardiology, The First Affiliated Hospital of Xinjiang Medical University

Objective To evaluate del transfection efficiency using recombinant adeno-associated virus Serotype 9 mediated enhanced green fluorescent protein (rAAV9- EGFP) to rats $\mathrm{H} 9 \mathrm{C} 2$ cells and the impact on growth of H9C2 cells.

Methods rAAV9-EGFP was transfected into H9C2 cells at different multiplicities of infection $(\mathrm{MOI}=1 \times 105,1 \times 106,1 \times 107)$. EGFP expression in the cells was observed under inverted fluorescence microscope and the EGFP-positive cell percentage determined by flow cytometry. Alamar Blue assay was used to assess the proliferation of the transfected cells.

Results The cells with rAAV9-EGFP transfection at MOI of $1 \times 106$ and $1 \times 107$ began to exhibit EGFP expression 1 days del after transfection and the cells transfection at MOI of $1 \times 105$ began to exhibit EGFP expression 2 days after transfection. The fluorescence intensity increased with the MOI used for transfection. EGFP expression reached the maximum on day 4 , at the point of which the transduction efficiency of rAAV9-EGFP in H9C2 cells was $(14.1 \pm 0.2) \%, \quad(35.1 \pm 4.8) \%$ and $(56.8 \pm 0.1) \%$. Corresponding to MOIs of $1 \times 105,1 \times 106$ and $1 \times 107$, respectively. Alamar Blue assay did not reveal significant difference in the absorbance between the transfected cells and the control cells after transfection.

Conclusions rAAV9-EGFP gene can be del transfected in a stable manner and efficiently expressed in H9C2 cells without causing cell growth inhibition. This del The results of this study played foundation (del) (provides a platform) for further research.

\section{e0055 RELATIONSHIP BETWEEN GENETIC POLYMORPHISM OF CYTOCHROME P4502C19 AND CORONARY ARTERY DISEASE IN XINJIANG UIGUR POPULATION}

doi:10.1136/hrt.2010.208967.55

Yang Yining, Ma Yitong, Wang Xinlei, Liu Fen, Xiang Xie. Department of Cardiology, The First Affiliated Hospital Xinjiang Medical University

Objective To study the relationship between cytochrome P450 family 2C19 subfamily genetic polymorphism and coronary artery disease in Xinjiang Uigur Population.

Methods There are (del) $370 \mathrm{CAD}$ patients and 190 control subjects, who (del) were tested for the genotypes for the (of) CYP2C19 SNPs rs4986893 with the methods (use) of PCR and analysed by restriction fragment length polymorphism. Blood press, (del) (,) plasma biochemical results and smoking were also included (del) (assessed). Statistic analyses were (del) (analysation was) performed with the SPSS 12.0 software package. The Hardy-Weinberg equilibrium was tested in all the groups.
Results Uigur, the genotypes distribution of the control group and the CHD group were in the Hardy-Weinberg equilibrium the (del), $\mathrm{p}>0.05$ (for case $\mathrm{p}=0.2$, for control $\mathrm{p}=0.068$ ). The frequency of $\mathrm{AA}$, AG and GG were $0.3 \%, 0.5 \%$ and $94.3 \%$ in the CAD group, compared with the control group that were $0.6 \%, 5.6 \%$ and $93.8 \%$ respectively. For $\mathrm{x}^{2}=0.107$, the $\mathrm{p}$ value was 0.743 . There was no significant difference in between the two groups. But the (amt?level???) blood glucose in the two groups had a difference (del). Smoking was also different in the CAD and the control groups $(p=0.0002)$.

Conclusions The distribution frequency of the CYP2C19 SNPs rs4986893 genotypes in the Uigur population with or without CAS is not significantly different with the blood glucose and smoking both have correlation with $\mathrm{CAD}$ (del).

\section{e0056 CORRELATION BETWEEN VIABLE MYOCARDIUM AND PZF IN SWINE MODEL OF ACUTE MYOCARDIAL INFARCTION}

doi:10.1136/hrt.2010.208967.56

Lin Tao, Ma Yi-Tong, Yang Yi-Ning, Mu Hu-Yati. Department of Cardiology, The First Affiliated Hospital, Xinjiang Medical University

Objective The aim of this study is to explore the relationship between the viable myocardium and Pzf of related coronary artery in swine model of acute myocardial.

Methods 13 swines, received coronary arteriongraphy after anaesthetic tracheal cannula, the pressure of coronary arteries were measured through guiding wire and the distal flow velocity of left anterior descending coronary arteries measured through doppler wire. Drawing the pressure- flow velocity coordinate graph, then calculated Pzf. Blocked up distal of left anterior descending coronary arteries by angioplasty balloon for 60-90 min to establish the model of acute myocardial infarction. The Pzf were calculated repeadly. Analysis the Pzf difference between before and after the model of acute myocardial infarction established. Taken out of those swines' hearts after executed them, sliced the cardiac muscle to dye with TTc to detect the viable myocardium, then cutted them from the left ventricular myocardium. The percent value of area viable myocardium were estimated by calculated the ratio of weight of viable myocardium to whole left venricular myocardium Drawing the percent value of area viable myocardium-Pzf after the model of acute myocardial infarction established coordinate graph and observed relationship between them.

Results 11 out of 13 swines were successfully established models of acute myocardial infarction by coronary occlusion. 2 swines died of entricular fibrillation after their coronaries were occluded 45 and 65 min respectively. The viable myocardium could be detected by using TTc dyeing. The result of statistics analysis confirmed that the percent value of area viable myocardium were negative correlation with Pzf. Conclusion The swine model of acute myocardial infarction can be established successfully by coronary occlusion with angioplasty balloon. The percent value of area viable myocardium were negative correlation with Pzf, which showed that Pzf was a new method to predict the area of viable myocardium after acute myocardial infarction.

\section{e0057 CORRELATION OF ABCA1 GENE R219K IN THE UIGHUR NATIONAL MINORITY IN XINJIANG WITH LIPIDMETABOLISM AND THE RISK OF MI}

doi:10.1136/hrt.2010.208967.57

Sun Minghui, Ma Yitong, Yang Yining. Department of Cardiology, The First Affiliated Hospital, Xinjiang Medical University

Objective To study the correlation of ATP binding cassette transporter $\mathrm{A} 1$ (ABCA1) and the gene polymorphism of $\mathrm{R} 219 \mathrm{~K}$ in the 